\title{
Chromosome territory repositioning induced by PHA-activation of lymphocytes: A 2D and 3D appraisal
}

\author{
Dimitrios loannou', Lakshmi Kandukuri ${ }^{1,2}$, Joe Leigh Simpson ${ }^{1}$ and Helen Ghislaine Tempest ${ }^{1,3^{*}}$
}

\begin{abstract}
Background: Genomes and by extension chromosome territories (CTs) in a variety of organisms exhibit nonrandom organization within interphase nuclei. CTs are susceptible to movement upon induction by a variety of stimuli, including: cell differentiation, growth factors, genotoxic agents, proliferating status, and stimulants that induce novel transcription profiles. These findings suggest nuclear architecture can undergo reorganization, providing support for a functional significance of $\mathrm{CT}$ organization. The effect of the initiation of transcription on global scale chromatin architecture has been underexplored. This study investigates the organization of all 24 human chromosomes in lymphocytes from two individuals in resting and phytohaemagglutinin activated lymphocytes using 2D and 3D approaches.
\end{abstract}

Results: The radial organization of CTs in lymphocytes in both resting and activated lymphocytes follows a gene-density pattern. However, CT organization in activated nuclei appears less constrained exhibiting a more random organization. We report differences in the spatial relationship between homologous and heterologous CTs in activated nuclei. In addition, a reproducible radial hierarchy of CTs was identified and evidence of a CT repositioning was observed in activated nuclei using both 2D and 3D approaches.

Conclusions: Alterations between resting and activated lymphocytes could be adaptation of CTs to the new transcription profile and possibly the formation of new neighborhoods of interest or interaction of CTs with nuclear landmarks. The increased distances between homologous and heterologous CTs in activated lymphocytes could be a reflection of a defensive mechanism to reduce potential interaction to prevent any structural chromosome abnormalities (e.g. translocations) as a result of DNA damage that increases during lymphocyte activation.

Keywords: Genome organization, Chromosome territories, Phytohaemagglutinin, Repositioning

\section{Background}

Genomes contain the blueprints of life and are organized in-vivo as chromosomes. Nonetheless, our understanding of the spatial organization of genomes and its function has received little attention compared with the vast body of knowledge of the majority of other cellular structures. Pioneering visualization experiments during the 1980s using fluorescence in situ hybridization (FISH) demonstrated that chromosomes were not randomly organized in mammalian cells, occupying distinct positions

\footnotetext{
* Correspondence: htempest@fiu.edu

'Department of Human and Molecular Genetics, Herbert Wertheim College of Medicine, Florida International University, Miami, FL 33199, USA

${ }^{3}$ Biomolecular Sciences Institute, Florida International University, Miami, FL 33199, USA

Full list of author information is available at the end of the article
}

known as chromosome territories (CTs) [1-6]. These CTs are roughly spherical in shape and $2-4 \mu \mathrm{m}$ in diameter [7]. Analyses using 3C technology have confirmed nonrandom organization of the genome with current evidence supporting a fractal globule organization of chromatin at all levels, from CTs to chromosome arm and band domains to megabase-sized chromatin built up from a series of spatially separated $100 \mathrm{kbp}$ chromatin domains [8]. Certain biophysical properties of the fractal globule (reviewed in [9]) further support this appealing model for chromatin organization.

Observations in different cell types and organisms identified proximity patterns of chromosomes, leading to the proposal of two models (gene density and chromosome size) for the radial arrangement of CTs. The gene density model proposes that gene-rich CTs and gene- 
dense subchromosomal regions are located toward the nuclear interior, with gene-poor regions located toward the nuclear periphery $[8,10,11]$. This model originated from observations in proliferating lymphoblasts and fibroblasts and can be seen in primates, old world monkey, rodents, birds (excluding chicken) and cattle [6, 12-14]. The chromosome size model originated from observations in flat ellipsoid fibroblasts, quiescent, and senescent cells proposing localization of larger chromosomes toward the nuclear periphery and smaller chromosomes toward the nuclear interior [15-17]. It is likely that these two models are not mutually exclusive, with radial CT organization depending on the proliferating status of the cells, the chromosome itself and the neighborhood surrounding it, which could play a vital role in regulating cell-type specific gene expression [18].

The above correlative observations of CTs and gene positioning have established the concept of nonrandom organization and highlight emphatically the question of whether there is a link between position and genome function. Evidence to support the hypothesis for a link between position and function is provided from studies of cellular differentiation processes. Examples include the repositioning of the immunoglobulin gene cluster, the Mash1 locus during neural induction [19, 20], the HoxB1 gene in mouse embryos [21], the repositioning of adipogenesis genes during porcine mesenchymal stem cell adipogenesis [22] and sex chromosome movement during porcine spermatogenesis [23]. These studies correlate gene repositioning (to a more internal localization) upon transcriptional activation (with the exception of the sex chromosome movement in pigs). However, internal positioning and activation of genes seems to be an oversimplification since biallelicaly expressed genes, RNA polymerase II sites and heterochromatin can be found throughout the nucleus [21, 24]. Further evidence of gene repositioning, relative to their respective CTs or to other nuclear structures such as transcription factories and splicing speckles also reveals a correlation with transcriptional activity [8]. This evidence comes from studies using active and inactive genes [25], immuneFISH approaches in Ikaros protein [26], 3D positional experiments of adenine nucleotide translocase genes on the $\mathrm{X}$ chromosome [27], immediate activation of Myc proto-oncogene in mouse B lymphocytes [28] and the distance between promyelotic leukemia loci and nuclear bodies that seems to correlate with transcriptional activity [29]. More recent evidence from $3 \mathrm{C}$ and $4 \mathrm{C}$ conformation experiments shows an association of actively transcribed Hox genes in a cluster compared to silent genes that are located in a different region and form part of the active cluster once they are activated [30]. Another emerging feature of genome organization that may play a major role in the control of gene expression is the intraorganization of chromosomes within the CTs in 3D space. This refers to loops that are being formed in order for regions of chromosomes to interact in cis (e.g. locus control region of the $\beta$-globin gene which acts as an enhancer of the $\beta$-globin genes), or trans (e.g. in mouse erythroid cells) [7]. The most prominent example of this intraorganization of CTs occurs during $\mathrm{X}$ chromosome inactivation in which the ncRNA Xist silences one of the $\mathrm{X}$ chromosomes in females, with only a handful of genes escaping inactivation. The $\mathrm{X}$ chromosome is inactivated by being condensed into a compact structure (Barr body) that is associated with the nuclear periphery. Following silencing, a repressive nuclear compartment forms that does not affect RNA polymerase II and transcription factors. Genes that are not expressed are "pulled" down into this repressive compartment, rendering themselves inaccessible to transcriptional machinery, whereas the few genes that escape inactivation and are expressed, loop out from this compartment [31]. The aforementioned examples highlight the relationship between the nonrandom organization of chromosomes and gene expression.

Another emerging aspect of the complex nature of genome organization arises from experiments in which the local environment changes by inducing differentiation or transcription through addition or removal of growth factors in cells that exist in culture, or through in-vitro exposure to genotoxic agents. Such experiments could be important in understanding the changes in genome organization as cells divide, age or reach the end of their replicative status. Evidence from primary fibroblasts that entered quiescence (after a 7 day serum starvation) depicts alterations in the topology of CTs including 13, 18 and 10 [16]. With chromosomes 13 and 18 exhibiting a movement toward the nuclear interior and chromosome 10 moving from an intermediate position to a more peripheral one [32]. A more detailed look at the gene expression of ten genes on chromosome 10 following this movement showed that two genes were down-regulated and five were up-regulated when CT 10 was in the periphery providing small scale evidence that the nuclear periphery is not solely associated with gene silencing [32]. Interestingly, the same group measured timing of the relocation of chromosomes after elimination of serum and remarkably CTs relocated within $15 \mathrm{~min}$, highlighting need for energy for this type of repositioning and implicating myosin $1 \beta$ as the mediator for this relocation [32]. In terms of cells in senescence, evidence from dermal fibroblasts demonstrates that CTs orient themselves following a size-related, rather than gene density related model [33]. These changes are another manifestation of the modification of nuclear architecture during these specific cellular stages that could lead to changes in the transcriptional status of the cells. Nuclear architecture is also subject to alterations when 
stimuli initiate cellular growth, transcriptional activation or induce DNA damage in vitro. The most prominent example for the former comes from pig mesenchymal stem cells when adipogenic growth factors added in culture give rise to committed pre-adipocyte cells. Six genes involved in the adipogenesis pathway repositioned to a more interior location after 14 days of treatment, correlated with up-regulation. The GATA2 gene moved from a peripheral to an interior location (day 7 - up-regulation) and back to a peripheral location (day 14 - downregulation) [32]. Recently, we reported reproducible events of CT repositioning in human lymphocytes following in-vitro exposure to genotoxic agents, hydrogen peroxide and UVB [20]. Differences were also reported in $\mathrm{CT}$ repositioning between the two genotoxic agents most likely represents differences in mobility and/or decondensation of CTs as a result of differences in the DNA damage induced, chromatin regions targeted and different repair mechanisms [20]. With regards to stimulating a different transcriptional profile a single study has provided evidence of CT repositioning in human lymphocytes following activation of lymphocytes using phytohaemagglutinin (PHA). PHA is a plant mitogen that induces the proliferation of mammalian lymphocytes and creates a cascade of biochemical events that activates resting lymphocytes, which results in large scale decondensation of chromatin, increases in nuclear size and leads to a distinct transcriptional profile [34]. Branco et al. [34] investigated the positioning of 11 CTs in resting human lymphocytes (-PHA) and activated human lymphocytes (+PHA) from a single female subject [34]. The findings showed some intraorganization of CTs with chromosomes 1 and 3 moving more peripherally and chromosome 21 being more centrally located in activated cells [34]. The differences between the two states were attributed to the nuclear expansion as a result of lymphocyte activation by PHA, and to the different transcriptional program [34]. Another important finding was the observation that CT intermingling was lower in activated lymphocytes. This finding was proposed to be a potential protective mechanism to prevent chromosome translocations or risk from DNA damage due to the controlled cell death program that occurs during $\mathrm{T}$ lymphocyte activation [34].

Altered transcription profiles induces changes in the topology of CTs; therefore, our study will expand on the previous PHA study [34] to investigate whether the organization of all 24 CTs differs between resting (-PHA) and activated (+PHA) human lymphocytes. Lymphocytes were thus obtained from two volunteers ( 1 male, 1 female) and CT organization for all 24 CTs was assessed utilizing both 2D and 3D approaches. The purpose of this study was to assess the topology of all 24 chromosomes in resting and transcriptionally activated lymphocytes and where possible compare 2D and 3D approaches. This study specifically investigates: i) random/nonrandom CT organization (2D); ii) spatial relationship between homologous CTs (intraprobe) and heterologous CT pairs (interprobe) (3D); iii) hierarchical organization of CTs from the nuclear interior toward the nuclear periphery (2D and 3D); and iv) CT repositioning in PHA activated lymphocytes (2D and 3D).

Findings in this study suggest that CT organization in lymphocytes is reproducible among subjects and follows a gene density pattern as identified by both $2 \mathrm{D}$ and $3 \mathrm{D}$ approaches. In addition, a number of alterations in CT organization was observed in activated lymphocytes compared to resting lymphocytes including: i) a less constrained CT organization (more random organization); ii) differences in the spatial organization of homologous and heterologous CTs; iii) small differences in the radial CT hierarchy; and iv) evidence of CT repositioning for a handful of CTs. When possible, comparisons between 2D and 3D approaches revealed largely similar results.

\section{Results}

Although both 2D and 3D approaches can be used to address the same problems, they utilize different approaches allowing different perspectives to be studied. Specifically, the 2D approach examines the radial distribution of the entire CT within a flattened 2D nucleus. The 3D approach utilizes the geometrical center of the CT (single point) and performs measurements in microns to the nearest nuclear edge or geometrical center of another $\mathrm{CT}$, providing a physical location or distance within a 3D nucleus.

\section{D radial chromosome territory organization in resting and activated lymphocytes}

The 2D organization of CTs within lymphocytes was assessed using previously published and validated methodologies that divide the interphase nucleus into five equal areas based on DAPI fluorescence intensity, and measures the fluorescence distribution of each CT across the five equal areas $[10,20,26,35]$. This provides the ability to determine if a CT is equally distributed across the nucleus (random organization), or demonstrates a preferential nuclear localization (nonrandom organization). A total of 9400 cells were captured and analyzed to assess the radial nuclear organization for all 24 human chromosomes in resting (-PHA) and activated (+PHA) lymphocytes. The CT distribution and random/nonrandom status of all 24 CTs is presented in Fig. 1 and Table 1, respectively, for both subjects and in both resting and activated lymphocytes. In resting lymphocytes, the vast majority of CTs occupied nonrandom positions ( $p<0.05), 22$ CTs $(91.67 \%)$ and 21 CTs $(91.30 \%)$, in the male and female subject respectively (Table 1). Nineteen CTs demonstrated nonrandom organization in both subjects (CT Y excluded). In activated lymphocytes a different picture of nuclear organization emerges, with CTs demonstrating a more 


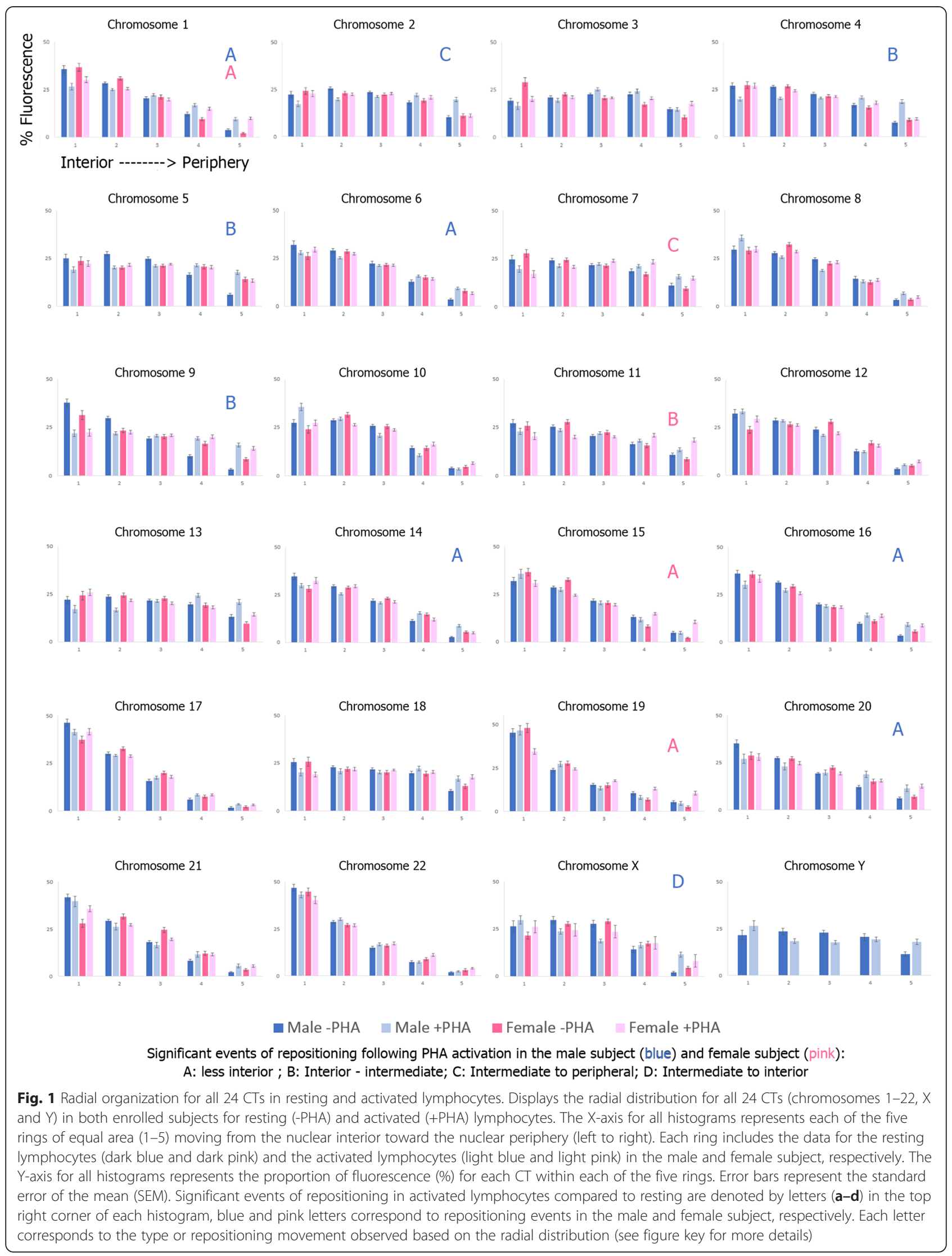


Table 1 Random/nonrandom organization status of all 24 CTs in $2 \mathrm{D}$ resting and activated lymphocytes

\begin{tabular}{|c|c|c|c|c|}
\hline \multirow[t]{2}{*}{$\mathrm{CT}$} & Male & Female & Male & Female \\
\hline & -PHA & -PHA & $+\mathrm{PHA}$ & $+\mathrm{PHA}$ \\
\hline 1 & NR & NR & NR & NR \\
\hline 2 & NR & NR & & NR \\
\hline 3 & & NR & & \\
\hline 4 & NR & NR & & NR \\
\hline 5 & NR & & & \\
\hline 6 & NR & NR & $N R$ & NR \\
\hline 7 & NR & NR & & \\
\hline 8 & $N R$ & $N R$ & $N R$ & $N R$ \\
\hline 9 & $N R$ & NR & & \\
\hline 10 & $N R$ & NR & $N R$ & NR \\
\hline 11 & NR & NR & & \\
\hline 12 & $N R$ & NR & $N R$ & $N R$ \\
\hline 13 & & NR & & \\
\hline 14 & $N R$ & $N R$ & $N R$ & $N R$ \\
\hline 15 & NR & NR & NR & $N R$ \\
\hline 16 & NR & NR & $N R$ & NR \\
\hline 17 & NR & $N R$ & $N R$ & NR \\
\hline 18 & NR & & & \\
\hline 19 & $N R$ & NR & NR & $N R$ \\
\hline 20 & NR & NR & $N R$ & NR \\
\hline 21 & NR & NR & NR & $N R$ \\
\hline 22 & $N R$ & $N R$ & $N R$ & $N R$ \\
\hline$x$ & $N R$ & $N R$ & $N R$ & $N R$ \\
\hline$Y^{*}$ & NR & - & & - \\
\hline
\end{tabular}

Presents the status (nonrandom or random) of CT organization for all chromosomes in resting (-PHA) and activated (+PHA) lymphocytes for each participant enrolled in this study. A minimum of 100 cells were captured and analyzed per $\mathrm{CT}$, per subject in both resting (-PHA) and activated (+PHA) lymphocytes. The $x^{2}$ goodness of fit test (d.f: 4 ) was utilized to determine whether the 2D radial distribution of each CT was random $(p>0.05)$ or nonrandom $(p<0.05)$. Nonrandom organization is denoted by NR $(p<0.05)$ random organization $(p>0.05)$ is denoted by blank boxes. * Data for the $Y$ chromosome is only available from the male subject

random organization compared to resting lymphocytes. In activated lymphocytes, 14 CTs $(58.33 \%)$ and 16 CTs (69.56\%) occupied a nonrandom organization in the male and female subject, respectively (Table 1). CTs that demonstrated a random organization in resting lymphocytes in the male and female subject (CTs 3 and 13; and CTs 5 and 18 , respectively) retained their random organization in activated lymphocytes. In the activated lymphocytes, an additional 8 CTs in the male subject and 5 CTs in the female subject were found to be randomly organized. Of the CTs that were randomly organized in the activated lymphocytes seven were common in both subjects (CTs 3, 5 , 7, 9, 11, 13 and 18), whereas CTs 2, 4 and Y were randomly organized only in the male subject.
Spatial relationship between CTs within the 3D nucleus Dual color 3D FISH experiments utilizing the same CT pairs permits the spatial relationship between homologous CTs (intraprobe) and between heterologous CTs probed together (interprobe) to be investigated. 3D models were rendered in Imaris (V.7.6.3) and the intraand interprobe measurements were established by measuring from the geometrical center of each CT between homologous CTs (intraprobe) and between heterologous CTs (interprobe) (Fig. 2). In addition, the 3D software also calculates the $3 \mathrm{D}$ volume of each rendered nucleus $\left(\mu \mathrm{m}^{3}\right)$. A minimum of 40 cells per CT were studied (20 per subject), with exceptions for the sex chromosomes because: i) no intraprobe probe measurements are available for the Y chromosome; ii) intraprobe probe measurements for the $\mathrm{X}$ chromosome are available from the female subject only (20 cells); and iii) interprobe measurements between the $\mathrm{X}$ and $\mathrm{Y}$ chromosome are available from the male subject only (20 cells). In Table 2 we report these results: i) CT pairs probed together, ii) intra- and interprobe measurements $(\mu \mathrm{m})$ for each CT, iii) nucleus diameter $(\mu \mathrm{m})$; and iv) nucleus volume $\left(\mu \mathrm{m}^{3}\right)$ in both resting and activated lymphocytes. The emerging picture from these data demonstrates that nuclei of activated lymphocytes were larger in size than that of resting lymphocytes (diameter and volume). In accordance with the increase in nucleus size, the intraand inter-probe distances were also greater for all CTs in activated lymphocytes compared to resting lymphocytes. Prior to comparing the intra- and interprobe data in both resting and activated lymphocytes, the measurements obtained were normalized against the diameter of the nucleus to account for differences in nucleus size (data not shown). Utilizing these normalized values, it is possible to order CTs based on their proximity (closest furthest). In resting lymphocytes the intraprobe measurements between homologous CTs (closest to furthest) were as follows: 19, 21, 22, 17, 1, 15, 16, 14, X, 10, 12, 6, $13,20,8,11,9,18,3,7,2,4$, and 5 . In activated lymphocytes the distances between homologous CTs (closest to furthest) were: $22, \mathrm{X}, 19,21,17,12,9,14,16,18,15,3,20$, $8,13,4,6,10,11,5,1,2$, and 7 . When the relative distances are compared between resting and activated lymphocytes, 9 CTs demonstrated the largest increases in distances in activated lymphocytes $(2,11,15$, $19,6,7,21,10$, and 1 , respectively). Conversely, CT X in activated lymphocytes in the female subject showed a closer spatial organization to that seen in resting lymphocytes. The normalized interprobe distances among CTs when probed together in resting lymphocytes (closest to furthest) were: $17-22,21-20,14-16,12-11,8-7$, $\mathrm{X}-\mathrm{Y}, 15-3,6-5,10-9,19-18,1-13$, and 2-4. In activated lymphocytes the spatial relationship between CT pairs (closest to furthest) were: 21-20, 12-11, 15-3, 19-18, 


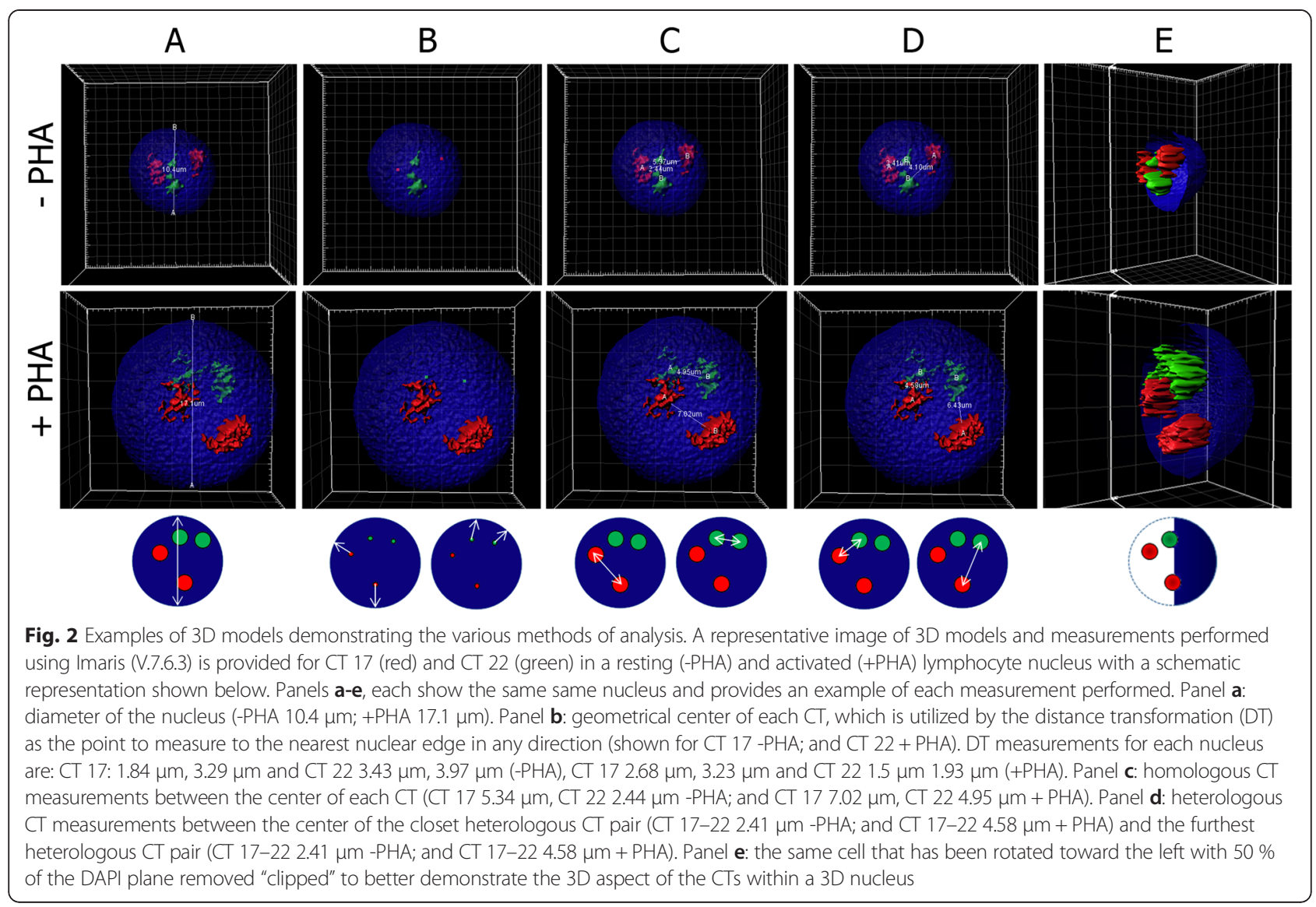

10-9, 14-16, 17-22, 6-5, 8-7, 1-13, 2-4 and X-Y. When comparing distances in activated lymphocytes compared to resting lymphocytes, five CT pairs demonstrated increasing distance $(6-5,14-16,17-22,8-7$, and $\mathrm{X}-\mathrm{Y}$, respectively) whereas, $7 \mathrm{CT}$ pairs demonstrated decreasing distance $(1-13,2-4,15-3,10-9,21-20,12-11$, and $19-18$, respectively).

\section{CT organization from the nuclear interior toward the nuclear periphery (2D and 3D approach)}

Our 2D method of analysis allows the distribution of CT fluorescence to be transformed to provide a single number for each nucleus, which reflects the midpoint of the frequency distribution of observed fluorescence (median) across the five rings [20]. This median value can be utilized to establish the hierarchical radial order of CTs from the nuclear interior toward the nuclear periphery [20]. The midpoint of the frequency distribution of the fluorescence for each CT in resting and activated lymphocytes was determined from a minimum of 200 cells per CT (100 cells per subject), with the exception of the CT Y, (studied in 100 cells from the male subject) (Table 3 ). In addition, hierarchical radial organization of CTs in resting and activated lymphocytes was also established from the 3D data. The hierarchical order of all 24 CTs was determined by the distance in microns, measured from the geographical center of the CT to the nearest nuclear edge. A minimum of 40 cells per CT (20 cells per subject) were analyzed with the exception of CT Y. To account for differences in nucleus size, the measurements obtained were normalized against the radius of the nucleus (Table 3). Despite inherent differences in the $2 \mathrm{D}$ and $3 \mathrm{D}$ analysis (2D: CT median fluorescence intensity; and 3D: distance from the CT geometrical center to nearest nuclear edge) the hierarchical organization of CTs in resting lymphocytes is by and large very similar between the two methodologies (Table 3). Both 2D and 3D methods show the same CTs forming clusters that compose the core, intermediate and peripheral regions of the nucleus: i) CTs: $1,15,16,17,19,21,22$; ii) CTs: 6,8 , 9, 10, 11, 12, 14, 20, X; and iii) CTs: 2, 3, 4, 5, 7, 13, 18 and Y (Table 3). The hierarchical organization of CTs in activated lymphocytes also demonstrates similar clustering; however, more variability in the clusters of CTs forming the core, intermediate and peripheral regions of the nucleus is observed between 2D and 3D methods (Table 3).

\section{CT repositioning following PHA activation of lymphocytes utilizing 2D and 3D approaches}

Furthermore, we evaluated whether there was any statistically significant alteration in the localization of CTs 
Table 2 Spatial relationship of CTS, nuclear diameter, and volume within 3D resting and activated lymphocytes

\begin{tabular}{|c|c|c|c|c|c|c|c|}
\hline CTs & $\begin{array}{l}\text { PHA } \\
\text { Status }\end{array}$ & $\begin{array}{l}\text { Intraprobe left CT } \\
\mu \mathrm{m}\end{array}$ & $\begin{array}{l}\text { Intraprobe right } \mathrm{CT} \\
\mu \mathrm{m}\end{array}$ & $\begin{array}{l}\text { Interprobe closest } \\
\mu \mathrm{m}\end{array}$ & $\begin{array}{l}\text { Interprobe furthest } \\
\mu \mathrm{m}\end{array}$ & $\begin{array}{l}\text { Nuclear diameter } \\
\mu m\end{array}$ & $\begin{array}{l}\text { Nuclear volume } \\
\mu \mathrm{m}^{3}\end{array}$ \\
\hline $1-13$ & $-\mathrm{PHA}$ & $4.21(1.07)$ & $4.79(1.28)$ & $3.52(1.75)$ & $5.70(2.0)$ & $10.27(0.98)$ & 996.17 (278) \\
\hline $1-13$ & $+\mathrm{PHA}$ & $10.32(2.53)$ & $8.82(4.31)$ & $5.48(2.26)$ & 9.59 (2.98) & $17.15(2.4)$ & $2922.92(1083)$ \\
\hline $2-4$ & $-\mathrm{PHA}$ & $5.83(2.06)$ & $5.99(1.66)$ & $3.72(1.57)$ & $5.89(1.87)$ & $10.54(0.88)$ & $964.10(137)$ \\
\hline $2-4$ & $+\mathrm{PHA}$ & $10.42(3.29)$ & 8.99 (2.78) & $5.01(2.19)$ & $9.76(3.22)$ & $16.65(2.07)$ & 3171.36 (1003) \\
\hline $15-3$ & $-\mathrm{PHA}$ & $4.49(1.11)$ & $5.47(1.95)$ & $2.80(1.42)$ & $5.11(1.87)$ & $10.45(1.02)$ & 893.98 (236) \\
\hline $15-3$ & $+\mathrm{PHA}$ & $8.62(2.7)$ & $8.71(2.81)$ & $4.00(2.2)$ & $8.48(2.63)$ & $17.23(2.21)$ & 3126.29 (1034) \\
\hline $6-5$ & $-\mathrm{PHA}$ & $4.89(1.64)$ & $6.11(2.03)$ & $2.86(1.28)$ & $5.13(1.59)$ & $10.49(1.11)$ & 815.17 (238) \\
\hline $6-5$ & $+\mathrm{PHA}$ & $8.74(3.23)$ & $9.48(2.67)$ & $4.31(2.32)$ & $8.80(2.92)$ & $15.93(1.95)$ & 2831.50 (849) \\
\hline $8-7$ & $-\mathrm{PHA}$ & $5.36(1.81)$ & $5.99(1.71)$ & $2.92(1.18)$ & $5.49(1.90)$ & $11.18(1.10)$ & $971.98(216)$ \\
\hline $8-7$ & $+\mathrm{PHA}$ & $7.73(3.47)$ & $9.45(2.84)$ & $4.91(2.66)$ & $8.32(2.58)$ & $15.10(2.25)$ & $2422.48(984)$ \\
\hline $10-9$ & $-\mathrm{PHA}$ & $5.08(1.82)$ & $5.65(2.03)$ & 3.47 (1.59) & $5.58(1.78)$ & $11.12(0.88)$ & $997.48(227)$ \\
\hline $10-9$ & $+\mathrm{PHA}$ & 8.79 (2.75) & $7.48(2.65)$ & $4.31(2.15)$ & 7.99 (2.72) & $15.89(1.99)$ & $2789.34(936)$ \\
\hline $12-11$ & $-\mathrm{PHA}$ & 4.87 (1.39) & $5.17(1.83)$ & $2.81(1.34)$ & $5.05(1.64)$ & $10.62(1.06)$ & $806.81(183)$ \\
\hline $12-11$ & $+\mathrm{PHA}$ & $7.24(2.67)$ & $8.95(3.14)$ & $3.51(2.12)$ & 7.87 (3.39) & $16.04(2.41)$ & 2628.15 (914) \\
\hline $14-16$ & $-\mathrm{PHA}$ & $4.58(1.52)$ & $4.55(1.62)$ & $3.00(1.59)$ & $4.45(1.48)$ & $10.48(0.99)$ & $758.17(181)$ \\
\hline $14-16$ & $+\mathrm{PHA}$ & $7.83(3.03)$ & $7.86(2.04)$ & $4.50(2.36)$ & 8.29 (3.03) & $16.40(2.16)$ & $2945.85(1034)$ \\
\hline $17-22$ & $-\mathrm{PHA}$ & $4.25(1.52)$ & $3.78(1.29)$ & $2.85(1.06)$ & $4.32(1.66)$ & $10.54(1.07)$ & 706.02 (173) \\
\hline $17-22$ & $+\mathrm{PHA}$ & $5.88(1.73)$ & $4.81(1.23)$ & $3.95(1.41)$ & $6.41(3.11)$ & $13.14(1.85)$ & $2040.62(757)$ \\
\hline $19-18$ & $-\mathrm{PHA}$ & $3.26(1.34)$ & $5.38(2.44)$ & $3.40(1.31)$ & $5.36(1.53)$ & $10.48(1.24)$ & 873.05 (295) \\
\hline $19-18$ & $+\mathrm{PHA}$ & $6.88(1.71)$ & $8.63(3.77)$ & $4.80(2.66)$ & $8.75(3.02)$ & $17.70(2.3)$ & 3333.78 (1151) \\
\hline $21-20$ & $-\mathrm{PHA}$ & $3.60(1.6)$ & $5.17(2.33)$ & $2.39(1.41)$ & $5.12(2.07)$ & $10.98(1.32)$ & 931.65 (361) \\
\hline $21-20$ & $+\mathrm{PHA}$ & $7.79(3.2)$ & $9.26(3.42)$ & $3.71(2.03)$ & 8.05 (3.72) & $18.31(2.61)$ & 3530.47 (1335) \\
\hline$X-Y$ & $-\mathrm{PHA}$ & - & - & 3.99 (1.72) & - & $10.51(1.07)$ & 797.52 (224) \\
\hline$X-Y$ & $+\mathrm{PHA}$ & - & - & 8.75 (3.69) & - & $17.87(1.84)$ & $4225.90(972)$ \\
\hline X & $-\mathrm{PHA}$ & $4.68(1.83)$ & - & - & - & $10.67(0.95)$ & 831.76 (197) \\
\hline$x$ & $+\mathrm{PHA}$ & $5.32(2.19)$ & - & - & - & $10.76(1.46)$ & $1292.68(480)$ \\
\hline
\end{tabular}

Provides an overview of all the 3D measurements obtained utilizing Imaris software (V7.6.3) for all 24 CTs in resting and activated lymphocytes. The CTs column indicates which CTs were probed together in the dual FISH experiment (e.g. 1 - 13 indicates chromosomes 1 and 13). PHA status indicates whether data is from resting (-PHA) or activated (+PHA) lymphocytes. The remaining columns present the average measurements in microns ( $\mu \mathrm{m}$ or $\mu \mathrm{m}^{3}$ ) obtained from a minimum of 40 cells ( 20 per subject with the exception of the $X-Y$ and $X-X$ data, with 20 cells from the male and female subject evaluated, respectively). Fig. 2 provides examples of how each distance was measured. The standard deviation of the measurements in all studied cells is provided in parentheses. The intraprobe columns provides the measurement between homologous CTs, the first intraprobe column provides the distance between the first CT listed in the CT column (e.g. CT 1), with the second intraprobe column providing the distance between the homologous CT pair for the second CT in the CT column (e.g. CT 13). The two interprobe columns provides the distances between the two heterologous CTs (e.g. CT 1 and CT 13), the first interprobe column presents the distance for the two closest CTs, with the second column presenting the data for the two furthest CTs. Subsequently, both the nuclear diameter and volume is provided

between resting and activated lymphocytes within each of the subjects enrolled. To evaluate 2D CT repositioning, the percentage of fluorescence distribution in each shell (Fig. 1) was compared between resting and activated lymphocytes in each of the two subjects. Alteration were deemed statistically significant when the $p$ value from the chi-squared goodness-of-fit comparison was less than 0.05 . In total there were 15 significant $\mathrm{CT}$ repositioning events (10 in the male subject, and 5 in the female subject) from resting to activated lymphocytes (Table 4). Based on the histograms produced from the radial analysis, movement of CTs was classified into the following categories: 1) interior to less interior, 2) interior to intermediate, 3) interior to periphery, 4) intermediate to periphery, 5) intermediate to interior (Fig. 1, Table 4). CT 1 was the only CT that demonstrated statistically significant repositioning in activated lymphocytes in both subjects, and also depicted the same type of movement (interior to less interior). Interestingly, the only CT that demonstrated a significant repositioning event toward the nuclear interior was that of CT X in the male subject. Rather than assessing the radial distribution of the entire CT in a 2D object, 3D methodologies provides physical localization of the center of each $\mathrm{CT}$ in a $3 \mathrm{D}$ nucleus to the nearest nuclear edge in $3 \mathrm{D}$ 
Table 3 Comparison of radial hierarchical organization of CTs between 2D and 3D methods

\begin{tabular}{|c|c|c|c|c|}
\hline & $\begin{array}{l}\text { CT hierarchy -PHA 2D } \\
\text { (median) }\end{array}$ & $\begin{array}{l}\text { CT hierarchy - PHA 3D } \\
\text { (distance) }\end{array}$ & $\begin{array}{l}\text { CT hierarchy + PHA 2D } \\
\text { (median) }\end{array}$ & $\begin{array}{l}\text { CT hierarchy + PHA 3D } \\
\text { (distance) }\end{array}$ \\
\hline \multirow[t]{23}{*}{ Nuclear Interior } & 19 (1.78) & $19(0.61)$ & 22 (1.99) & $22(0.52)$ \\
\hline & $17(1.85)$ & $22(0.57)$ & $17(2.00)$ & $19(0.51)$ \\
\hline & $22(1.85)$ & $1(0.50)$ & 19 (2.06) & $21(0.48)$ \\
\hline & $1(2.08)$ & $15(0.49)$ & $21(2.14)$ & $17(0.46)$ \\
\hline & $21(2.10)$ & $17(0.49)$ & $10(2.27)$ & $16(0.42)$ \\
\hline & $16(2.12)$ & $16(0.48)$ & $15(2.29)$ & $1(0.41)$ \\
\hline & $15(2.17)$ & $21(0.46)$ & $8(2.31)$ & $12(0.41)$ \\
\hline & $9(2.23)$ & $20(0.45)$ & $14(2.34)$ & $15(0.40)$ \\
\hline & $14(2.25)$ & $12(0.44)$ & $16(2.34)$ & $8(0.40)$ \\
\hline & $20(2.30)$ & $14(0.43)$ & $12(2.37)$ & $10(0.39)$ \\
\hline & $8(2.33)$ & $X(0.43)$ & $6(2.49)$ & $7(0.39)$ \\
\hline & $6(2.34)$ & $8(0.42)$ & $X(2.53)$ & $20(0.39)$ \\
\hline & $12(2.34)$ & $6(0.42)$ & $1(2.54)$ & $14(0.39)$ \\
\hline & $10(2.38)$ & $11(0.40)$ & $20(2.63)$ & $6(0.38)$ \\
\hline & $X(2.42)$ & $10(0.40)$ & $4(2.76)$ & $9(0.38)$ \\
\hline & $11(2.49)$ & $9(0.39)$ & $5(2.85)$ & $Y^{*}(0.36)$ \\
\hline & $4(2.53)$ & $2(0.37)$ & $9(2.89)$ & $18(0.35)$ \\
\hline & $7(2.58)$ & $Y^{*}(0.37)$ & $2(2.96)$ & $X(0.34)$ \\
\hline & $5(2.62)$ & $4(0.37)$ & $11(2.96)$ & $3(0.34)$ \\
\hline & $2(2.69)$ & $7(0.37)$ & $Y^{*}(2.98)$ & $13(0.33)$ \\
\hline & $13(2.73)$ & $13(0.34)$ & $3(3.04)$ & $5(0.33)$ \\
\hline & $3(2.77)$ & $3(0.34)$ & $18(3.04)$ & $4(0.32)$ \\
\hline & $18(2.77)$ & $18(0.32)$ & $13(3.05)$ & $2(0.32)$ \\
\hline Nuclear Periphery & $Y^{*}(2.83)$ & $5(0.32)$ & $7(3.12)$ & $11(0.31)$ \\
\hline
\end{tabular}

Presents the radial hierarchy for all 24 CTs in resting and activated lymphocytes using both $2 \mathrm{D}$ and $3 \mathrm{D}$ approaches from both subjects. CTs are ordered from the nuclear interior (top of the table) toward the nuclear periphery (bottom of the table). Following the CTs in parentheses are the numerical values used to order the CTs. In the 2D approach, CTs are ordered based on the midpoint of the distribution of the CT fluorescence across the five rings of equal area (median) (200 cells/ $\mathrm{CT}$ ). In the 3D approach, CTs are ordered based on their (distance) to the nearest nuclear edge following normalization against the radius of the nucleus to account for differences in nucleus size (40 cells/CT). Data for CT Y $\left(\mathrm{Y}^{*}\right)$ is obtained from the male subject only (2D: 100 cells; 3D: 20 cells)

models rendered in Imaris (V.7.6.3). As with the 2D analysis the nuclear localization of all 24 CTs was established in the same subjects in resting and activated lymphocytes. For the $3 \mathrm{D}$ analysis $\mathrm{CT}$ repositioning was established using a two-tailed, paired $t$-test $(p<0.05)$ to detect significant events of CT repositioning following activation of lymphocytes. In total 10 repositioning events were found to be statistically significant, six in the male subject (CTs $11,14,15,16,17$, and 19) and four in the female subject (CTs 11, 16, 19, and X) (Table 4). All significant CT movements in activated lymphocytes were relatively more peripheral compared to resting lymphocytes. Three CTs were common between the two subjects (CTs 11, 16, and 19), whereas two CTs were common between the two methods in the male (CTs 14 and 16), and in the female (CTs 11 and 19), all demonstrating a similar peripheral movement.

\section{Discussion}

The effects of transcriptional reprogramming on nuclear organization have been underexplored. Therefore the purpose of this study was to evaluate the consequences of PHA activation on global nuclear organization of CTs in human lymphocytes. To the best of our knowledge this is the first study to evaluate the radial organization, random/ nonrandom status, intra- and interprobe spatial relationship between CTs, radial hierarchy and repositioning of all 24 chromosomes in resting and PHA-activated lymphocytes using both $2 \mathrm{D}$ and $3 \mathrm{D}$ approaches in multiple subjects.

The $2 \mathrm{D}$ radial organization of CTs demonstrates a highly reproducible radial CT organization with CTs displaying similar radial distributions and random/nonrandom organization between subjects in both resting and 
Table 4 CTs exhibiting radial repositioning in activated lymphocytes compared to resting lymphocytes

\begin{tabular}{|c|c|c|c|}
\hline \multicolumn{4}{|l|}{ 2D repositioning } \\
\hline Male Subject & & Female Subject & \\
\hline CTs & Movement & CTs & Movement \\
\hline $1,6,14,16,20$ & Less interior & $1,15,19$ & Less interior \\
\hline $4,5,9$ & Interior to intermediate & 11 & Interior to intermediate \\
\hline 2 & Intermediate to periphery & 7 & Interior to periphery \\
\hline$x$ & Intermediate to interior & - & - \\
\hline \multicolumn{4}{|l|}{ 3D repositioning } \\
\hline Male Subject & & Female Subject & \\
\hline CTs & Movement & CTs & Movement \\
\hline $11,14,15,16,17,19$ & More peripheral & $11,16,19, x$ & More peripheral \\
\hline
\end{tabular}

CTs involved in statistical significant radial repositioning between resting and activated lymphocytes in both the male and female subject, as determined by $2 \mathrm{D}$ and $3 \mathrm{D}$ approaches. 2D radial repositioning was determined using the $x^{2}$ goodness-of-fit test $(p<0.05)$. The direction of the repositioning movement was determined by comparing the radial distribution of CTs in each subject in resting and activated lymphocytes (Fig. 1). 3D radial repositioning was determined using the two-tailed, paired $t$-test $(p<0.05)$. The direction of the repositioning movement was determined based on the distance to the nuclear edge in activated lymphocytes compared to resting lymphocytes

activated lymphocytes. Overall, the organization in activated lymphocytes appears to be more "relaxed" (or less defined), with more CTs (10 in the male subject and 7 in the female subject) displaying a random organization compared to resting lymphocytes (2 CTs in both subjects). It has been established that following PHA stimulation in human lymphocytes, there is a rapid transcriptional surge, which is accompanied by nuclear and nucleolar morphological changes [34]. Specifically, there is enlargement of the nuclear size, increased chromatin decondensation and activation of nucleoli [34]. In agreement with previously published studies, the current study also reports an increase in nuclear size $(\sim 2-3$ fold) in activated lymphocytes compared to resting lymphocytes [34]. The increased nuclear size in activated lymphocytes could account for the more random CT organization observed. Chromatin is a dynamic entity in constant motion inside the nucleus [36, 37]; thus, in a nucleus with more available space, it is feasible that there is relatively more motion at least for some CTs, perhaps conferring a less defined organization than other CTs.

Dual color 3D FISH experiments also enabled the spatial relationship between CTs to be examined, providing information on interactions amongst homologous chromosomes and heterologous CT pairs. Differences in nuclear size is a potential confounder, therefore, to account for these differences the distances were normalized using the nuclear diameter. The distances between homologous CTs (intraprobe) were larger in $74 \%$ (17 heterologous homologs) of cases in activated lymphocytes (CT Y excluded), with only 6 CTs possessing shorter distances. The interprobe results demonstrate that $58 \%$ of CTs (14 CTs) had relatively smaller distances, with $42 \%$ of CTs (10 CTs) possessing greater distances in activated lymphocytes compared to resting lymphocytes. In activated lymphocytes the following CT pairs demonstrated the largest increases in distance compared to resting lymphocytes: $17-22,8-7$ and $\mathrm{X}-\mathrm{Y}$ (male subject only). Altered spatial organization between homologous CTs could be due to different patterns of chromatin decondensation (or condensation) amongst different CTs, or as result of the new transcriptional profile that occurs following PHA activation [34]. The spatial relationship between CTs may also be the result of the formation of new CT neighborhoods following transcriptional activation, or serve as a protective mechanism to reduce potential interactions to prevent any structural abnormalities (e.g. translocations) as result of DNA damage that increases during $\mathrm{T}$ lymphocyte activation [34, 38].

Utilizing both 2D and 3D methodologies the radial hierarchy of all CTs from the nuclear interior toward the nuclear periphery was established. The radial organization of CTs in both resting and activated lymphocytes in this study follows a more gene-density correlation, which is in agreement with previously published literature $[4,11,15,20]$. This type of organization might be stimulated by chromosome specific gene expression and the organization of transcription in the nucleus (i.e. interaction of polymerases with CT physical properties like NORs) [16, 39]. Both 2D and $3 \mathrm{D}$ approaches provided remarkably similar results in terms of the hierarchical order of CTs that preferentially formed clusters in the nuclear interior, intermediate and peripheral regions. Gene rich CTs (e.g. CTs 15, 17, 19, and 22) clustered toward the nuclear interior, whereas gene poor CTs (e.g. CTs 2, 3, 13, and 18) localized toward the nuclear periphery in resting and activated lymphocytes. CT 21 is the only gene poor chromosome associated with the nuclear interior [40], a finding mirrored in a recently 
published study [20]. The intermediate region of the nucleus is largely composed of the following CTs: $6,8,10$, $12,14,20$, and X, of these, four CTs were of intermediate gene density (CTs 6, 10, 14, and X), two were gene rich (CTs 12 and 20) and one was gene poor (CT 8) [40].

$2 \mathrm{D}$ and $3 \mathrm{D}$ approaches were also utilized to evaluate any statistically significant events of CT repositioning between resting and activated lymphocytes. Both methods identified significant repositioning events with similarities emerging between the two approaches. These findings include more CTs repositioned in the male subject (e.g. 10 events, 2D and 6 events, 3D) compared to the female subject ( 5 events, 2D, and 4 events, 3D), and all CTs demonstrating a relatively more peripheral organization, with the exception of CT X (2D in the male subject). One repositioned CT (CT 1) was shared between the subjects in the $2 \mathrm{D}$ results with three repositioned CTs shared between the subjects using the 3D method (CTs 11, 16, and 19). When an intra-subject comparison was made between the two approaches (2D and 3D), CTs 14, and 16 and CTs 11 and 19 showed similar characteristics in the male and female subject, respectively. One possible explanation for the differences observed in the intra-subject comparisons regarding the number of CT repositioning events and the CTs involved could be due to variability in the nuclear size between the subjects. A two-tailed, paired $t$-test showed that in certain CTs (CTs 2, 4, 5, 6, 9, 10, 14, 16, 17, 22, $\mathrm{X}$ and $\mathrm{Y})$ there was a significant difference $(p<0.05)$ in the nucleus size between the male and female subject activated lymphocytes (data not shown). Interestingly, 8 out of the 10 significant events of CT repositioning involved these CTs. A similar $t$-test in resting lymphocytes only revealed a significant difference in nucleus size between the two subjects for CTs 3 and 15 depicting more comparable nuclei sizes (data not shown). The differences in the radial positions could reflect the different network interactions of chromosomes with other nuclear structures or alternative territories [34]. To date, one other study has investigated the repositioning of $11 \mathrm{CTs}$ (CTs 1, 2, 3, 4, 5, 9, 11, 12, 13, 21 and 22) in resting and activated lymphocytes [34]. This study, reported peripheral repositioning of CTs 1 and 3 and more internal repositioning of CT 21 [34]. The majority of repositioned CTs in the current study were not evaluated in the previous study, however, we confirm the peripheral repositioning of CT 1 but not for CT3, and although not significant in this study the radial hierarchy of CT 21 is more internally localized in activated lymphocytes.

An additional important aspect in the current study is the use of both $2 \mathrm{D}$ and $3 \mathrm{D}$ approaches to study CT organization. The two approaches to address CT organization are synergistic, the $2 \mathrm{D}$ approach assesses the radial distribution of the entire $\mathrm{CT}$ in a $2 \mathrm{D}$ nucleus, whereas with the 3D approach assesses the physical localization of the center of the CT to the nearest nuclear edge or another CT. Despite the inherent differences between the two approaches the relatively small variations observed in the hierarchical organization of CTs are reassuring, suggesting reproducibility between both $2 \mathrm{D}$ and $3 \mathrm{D}$ methods. The reproducibility between the two approaches is likely due to the fact that the $2 \mathrm{D}$ data was transformed to a single data point, similar to the 3D data, rather than examining the distribution of the entire CT. Greater variability was observed between the two approaches when examining $\mathrm{CT}$ repositioning in activated lymphocytes, most likely reflecting differences when studying localization of the entire CT (2D) versus a single point (3D). Nevertheless, some CTs demonstrated reproducible repositioning using both methods which provides further evidence that both 2D and 3D approaches provide comparable data [32].

\section{Conclusions}

Alterations observed in CT organization in activated lymphocytes most likely depend on and influence the spatial organization of CTs [34]. Both 2D and 3D approaches revealed CT specific differences in organization between resting and activated lymphocytes. These differences were associated with the increased nuclear size and altered chromatin condensation observed in activated cells, and may be related to the altered transcriptional profile in activated cells. Different CT interactions (changes in intra- and interprobe spatial organization) may lead to the formation of different CT neighborhoods. Furthermore, there is evidence to indicate that CTs in activated cells display less intermingling which may serve as a mechanism to reduce the formation of chromosome aberrations [34]. These hypotheses warrant further investigation in future studies focusing on the spatial relationship and interaction between CTs. In addition, future studies should include a larger cohort and genome-wide gene expression studies to determine whether consistency is observed among individuals for CT repositioning events and whether repositioning is associated with gene expression.

\section{Methods}

\section{Sample cohort}

This research study was approved by the Florida International University Institutional Review Board (protocol numbers: IRB-121010-00, IRB-14-0163). Informed consent was provided by two individuals (one male and one female subject) to participate in this study. Both participants were 35-years-old, karyotypically normal (data not shown) and completed a brief health history survey, providing life style (e.g. alcohol or tobacco use) any recent illness information, and any medication taken. Both 
participants were non-smokers and had not knowingly been in contact with any hazardous or radioactive material in their working or home environment and were occasional social drinkers (2-8 units/week).

\section{Conditions of Cell Culture}

Peripheral blood was collected by venipuncture in heparin tubes (Greiner-BioOne, Monroe, NC, USA). Whole blood from each individual was split and cultured in the presence or absence of PHA. All lymphocyte cultures were prepared in RPMI 1640 (Lonza, Walkersville, MD, USA) reconstituted with: $10 \%$ heat inactivated fetal bovine serum (FBS - Sigma-Aldrich, St Louis, MO, USA), $2 \%$ L-glutamine (Thermo-Fisher, Waltham, MA, USA) and $1 \%$ penicillin-streptomycin solution (Thermo-Fisher, Waltham, MA, USA). All cultures had a total volume of $5 \mathrm{ml}$ and for those that PHA was added, they were reconstituted with $100 \mu \mathrm{l}$ of PHA, (45 mg/vial) (Remel Inc, Lenexa, KS, USA) $0.8-1.0 \mathrm{ml}$ of blood was incubated for $71 \mathrm{~h}$ at $37^{\circ} \mathrm{C}\left(5 \% \mathrm{CO}_{2}\right)$. Following lymphocyte culture incubation $(71 \mathrm{~h})$, lymphocytes were prepared following standard karyotyping protocols. Proliferating cells in metaphase were arrested using $0.2 \mu \mathrm{g}$ colcemid (ThermoFisher, Waltham, MA, USA) for $30 \mathrm{~min}$ at $37^{\circ} \mathrm{C}$, followed by standard hypotonic conditions to allow separation of white blood cells from anucleate erythrocytes $(0.075 \mathrm{M}$ of KCL - Thermo-Fisher, Waltham, MA, USA) for $45 \mathrm{~min}$ at $37^{\circ} \mathrm{C}$. White blood cells were subsequently fixed in $3: 1(\mathrm{v} / \mathrm{v})$ of methanol:acetic acid solution to clean and fix the preparation. All cultures were stored at $-20{ }^{\circ} \mathrm{C}$ immediately following the harvesting procedure.

\section{Fluorescence in situ hybridization (FISH)}

Cells cultured in the presence and absence of PHA were dropped on glass slides (FisherBrand ${ }^{\circ}$ - Thermo-Fisher, Waltham, MA, USA), allowed to adhere by ageing overnight at room temperature (RT) and subsequently washed in 1X PBS (Thermo-Fisher, Waltham, MA, USA), followed by an ethanol dehydration step (70-80$100 \%, 3$ min each). Air dried cells were treated with a $1 \%$ pepsin solution (Thermo-Fisher, Waltham, MA, USA) in a pre-warmed at $37^{\circ} \mathrm{C}$ solution of $49 \mathrm{ml}$ double distilled water $\left(\mathrm{ddH}_{2} \mathrm{O}\right)$ and $0.5 \mathrm{ml}$ of $1 \mathrm{~N}$ HCL (Thermo-Fisher, Waltham, MA, USA) for $20 \mathrm{~min}$. Cells were rinsed with $\mathrm{ddH}_{2} \mathrm{O}$ and $1 \mathrm{X}$ PBS at RT, and subjected to another round of fixation using $1 \%$ paraformaldehyde/PBS (Thermo-Fisher, Waltham, MA, USA) at $4{ }^{\circ} \mathrm{C}$ for $10 \mathrm{~min}$. Following which, slides were rinsed in 1 $X$ PBS and $\mathrm{ddH}_{2} \mathrm{O}$ (RT), prior to another ethanol dehydration series (2 min each), and finally air dried. A dual color FISH experiment (fluorescein isothiocyanate (FITC), and tetramethyl rhodamine isothiocyanate (TRITC) labelled FISH probes) was performed utilizing whole chromosome paints (WCPs), for all 24 chromosomes
(Rainbow Scientific, Windsor, CT, USA). WCPs were codenatured for $5 \mathrm{~min}$ with lymphocytes at $75{ }^{\circ} \mathrm{C}$ followed by overnight hybridization $(>16 \mathrm{~h})$ at $37^{\circ} \mathrm{C}$ using a Thermobrite ${ }^{\circ}$ Statspin (Abbott Molecular, Illinois, IL, USA). A post hybridization stringency wash was performed in a pre-warmed $73{ }^{\circ} \mathrm{C}$ solution of $0.7 \mathrm{X} \mathrm{SSC} / 0.3 \%$ Tween 20 (Thermo-Fisher, Waltham, MA, USA) ( $35 \mathrm{ml}$ of $20 \mathrm{X}$ SSC, $3 \mathrm{ml}$ of Tween 20 and $965 \mathrm{ml}$ of $\mathrm{ddd}_{2} \mathrm{O}$ ) for $2 \mathrm{~min}$. After 2 min elapsed, cells were washed in $2 \mathrm{X} \mathrm{SSC/}$ $0.1 \%$ Tween $20(100 \mathrm{ml}$ of $20 \mathrm{X}$ SSC followed by a brief ethanol series ( $1 \mathrm{~min}$ each). Slides were subsequently air dried and mounted with 4',6-diamidino-2-phenylindole (DAPI - Vector Labs, Burlingame, CA, USA) under a 24X55mm coverslip.

\section{D Image acquisition, radial chromosome positioning and statistical analysis}

All images for 2D analysis were captured using an Olympus BX61 epifluorescence microscope equipped with a cool charged couple device camera (Hamamatsu ORCA $-\mathrm{R}_{2}$ C10600) and a motorized ES111 Optiscan stage (Prior Scientific UK). Three single band pass filters for FITC, TRITC, and DAPI (Chroma Technology, Bellows Falls, VT, USA) were used. All images were acquired using Smart Capture 3.0 (Digital Scientific, UK) exported as .tiff files for further analysis. A minimum of 100 cells were analyzed per subject, per chromosome pair, per condition (-PHA and + PHA). To evaluate the radial chromosome position previously published methodologies were utilized $[11,20]$. The details have been described extensively elsewhere $[20,26,28]$. In brief, a customized script written for Image J allows the separation of each captured image into three channels (FITC, TRITC and DAPI counterstain). The DAPI fluorescence is converted into a binary mask that allows for the creation of 5 concentric rings of equal area from nuclear interior toward the nuclear periphery. The proportion of WCP signal in each ring, for each channel (FITC and TRITC) is subsequently measured relative to the total signal for the area contained within the ring. Data was normalized against the different DNA content in the nucleus to compensate for the fact that a $3 \mathrm{D}$ object is observed in 2D. The Chi-squared goodness of fit $\left(\chi^{2}\right)$ was utilized to evaluate if the organization of each chromosome differed from random $(p<0.05)$ and to compare differences between conditions (-PHA and + PHA) in each subject.

\section{D Image acquisition, chromosome territory positioning and statistical analysis}

The FISH protocol described above was used to prepare slides for 3D image acquisition. The images were captured using a DeltaVision (Applied Precision, WA, USA) imaging station consisting of an Olympus IX71 inverted microscope with $\mathrm{x} 100,1.4$ NA oil-immersion lens and a 
photometric CCD. All images were taken with a Z step size of $1.0 \mu \mathrm{m}$ (36 optical sections), saved as 3D stacks and subjected to constrained iterative deconvolution (using DeltaVision - SoftWoRx -version 5.5 - software package - Applied Precision, WA, USA). A TRITC $(594 \mathrm{~nm})$, FITC (523 nm) and DAPI (435 nm) filter were used to capture the images and a minimum 20 images per CT, per subject, per condition (-PHA and + PHA) were captured. Images were exported as $3 \mathrm{D}$ stacks to Imaris software (V.7.6.3 Bitplane - Zurich, Switzerland) to perform 3D model reconstruction for each of the captured images and further analysis. The embedded distance transformation (DT) tool was used to calculate the distance from the center of the $\mathrm{CT}$ (geometric center) to the nearest nuclear edge of the reconstructed $3 \mathrm{D}$ model image. In addition, distances from the center of each $\mathrm{CT}$ were measured between homologous CTs (intraprobe) and heterologous CT pairs (interprobe). The measurements were normalized against the radius of the nucleus to account for differences in the sizes of each nuclei and the nucleus volume was calculated in Imaris. A twotailed paired $t$-test $(p<0.05)$ was used to evaluate any statistical significant differences in the repositioning of CTs (-PHA and + PHA) and to evaluate significant differences between intra- and interprobe distances and the volume of the nuclei.

\section{Abbreviations}

2D: 2 dimensions; 3D: 3 dimensions; CCD: Charge Couple Device; CT(s): Chromosome Territory (ies); DAPI: 4',6-diamidino-2-phenylindole; $\mathrm{ddH}_{2} \mathrm{O}$ : Double distilled water; DT: Distance transformation; FISH: Fluorescent in situ hybridization; FITC: Fluorescein isothiocyanate; PBS: Phosphate buffer saline; PHA: Phytohaemagglutinin; RT: Room temperature; SSC: Saline sodium citrate; WCP: Whole chromosome paint.

\section{Competing interests}

The authors declare that they have no competing interests.

\section{Authors' contributions}

$\mathrm{DI}$ - performed experiments (2D and 3D), data and statistical analysis, drafted manuscript; LK - performed 2D experiment \& culturing of lymphocyte cultures; JLS - experimental design, critical review of manuscript; HGT - conceived the project, assisted with data analysis, critical review of manuscript. All authors read and approved the final manuscript.

\section{Acknowledgements}

This study was funded in part by a Department of Defense grant "Mass Scale Biosensor Threat Diagnostic for In-Theater Defense Utilization" W81XWh-101-0732 and supported the following authors DI, LK, JLS and HGT. The authors would also like to acknowledge the financial support for this study provided by the Herbert Wertheim College of Medicine at Florida International University (startup funds awarded to HGT). The funders had no role in study design, data collection and analysis, decision to publish, or preparation of the manuscript.

\section{Author details}

'Department of Human and Molecular Genetics, Herbert Wertheim College of Medicine, Florida International University, Miami, FL 33199, USA. ${ }^{2}$ Present address Centre for Cellular and Molecular Biology (CCMB), Council of Scientific and Industrial Research (CSIR) Uppal Road, Hyderabad 500 007, India. ${ }^{3}$ Biomolecular Sciences Institute, Florida International University, Miami, FL 33199, USA.
Received: 15 April 2015 Accepted: 14 May 2015

Published online: 03 July 2015

\section{References}

1. Khalil A, Grant JL, Caddle LB, Atzema E, Mills KD, Arneodo A. Chromosome territories have a highly nonspherical morphology and nonrandom positioning. Chromosome Res. 2007;15:899-916.

2. Manuelidis L. A view of interphase chromosomes. Science. 1990;250:1533-40.

3. Oliver B, Misteli T. A non-random walk through the genome. Genome Biol. 2005;6:214.

4. Cremer M, von Hase J, Volm T, Brero A, Kreth $G$, Walter J, et al. Non-random radial higher-order chromatin arrangements in nuclei of diploid human cells. Chromosome Res. 2001;9:541-67.

5. Cremer T, Cremer C. Chromosome territories, nuclear architecture and gene regulation in mammalian cells. Nat Rev Genet. 2001;2:292-301.

6. Cremer T, Cremer M. Chromosome territories. Cold Spring Harb Perspect Biol. 2010;2:a003889.

7. Cavalli G, Misteli T. Functional implications of genome topology. Nat Struct Mol Biol. 2013;20:290-9.

8. Kolbl AC, Weigl D, Mulaw M, Thormeyer T, Bohlander SK, Cremer T, et al. The radial nuclear positioning of genes correlates with features of megabase-sized chromatin domains. Chromosome Res. 2012;20:735-52.

9. Mirny LA. The fractal globule as a model of chromatin architecture in the cell. Chromosom Res. 2011;19:37-51.

10. Boyle S, Gilchrist S, Bridger JM, Mahy NL, Ellis JA, Bickmore WA. The spatial organization of human chromosomes within the nuclei of normal and emerin-mutant cells. Hum Mol Genet. 2001;10:211-9.

11. Croft JA, Bridger JM, Boyle S, Perry P, Teague P, Bickmore WA. Differences in the localization and morphology of chromosomes in the human nucleus. J Cell Biol. 1999;145:1119-31.

12. Tanabe H, Kupper K, Ishida T, Neusser M, Mizusawa H. Inter- and intra-specific gene-density-correlated radial chromosome territory arrangements are conserved in Old World monkeys. Cytogenet Genome Res. 2005;108:255-61.

13. Tanabe H, Muller S, Neusser M, von Hase J, Calcagno E, Cremer M, et al. Evolutionary conservation of chromosome territory arrangements in cell nuclei from higher primates. Proc Natl Acad Sci U S A. 2002;99:4424-9.

14. Koehler D, Zakhartchenko V, Froenicke L, Stone G, Stanyon R, Wolf E, et al. Changes of higher order chromatin arrangements during major genome activation in bovine preimplantation embryos. Exp Cell Res. 2009;315:2053-63.

15. Bolzer A, Kreth G, Solovei I, Koehler D, Saracoglu K, Fauth C, et al. Three-dimensional maps of all chromosomes in human male fibroblast nuclei and prometaphase rosettes. PLoS Biol. 2005:3:e157.

16. Bridger JM, Boyle S, Kill IR, Bickmore WA. Re-modelling of nuclear architecture in quiescent and senescent human fibroblasts. Curr Biol. 2000;10:149-52.

17. Foster HA, Bridger JM. The genome and the nucleus: a marriage made by evolution. Genome organisation and nuclear architecture. Chromosoma. 2005; 114:212-29.

18. Cavalli G. Chromosome kissing. Curr Opin Genet Dev. 2007;17:443-50.

19. Schneider R, Grosschedl R. Dynamics and interplay of nuclear architecture, genome organization, and gene expression. Genes Dev. 2007;21:3027-43.

20. loannou D, Kandukuri L, Quadri A, Becerra V, Simpson JL, Tempest HG. Spatial Positioning of All 24 Chromosomes in the Lymphocytes of Six Subjects: Evidence of Reproducible Positioning and Spatial Repositioning following DNA Damage with Hydrogen Peroxide and Ultraviolet B. PLoS One. 2015;10, e0118886.

21. Takizawa T, Meaburn KJ, Misteli T. The meaning of gene positioning. Cell. 2008;135:9-13.

22. Szczerbal I, Foster HA, Bridger JM. The spatial repositioning of adipogenesis genes is correlated with their expression status in a porcine mesenchymal stem cell adipogenesis model system. Chromosoma. 2009;118:647-63.

23. Foster HA, Abeydeera LR, Griffin DK, Bridger JM. Non-random chromosome positioning in mammalian sperm nuclei, with migration of the sex chromosomes during late spermatogenesis. J Cell Sci. 2005;118:1811-20.

24. Yuan H, Cheng G, You L, Li H, Zhu H, Li W, et al. Influence of metal-MoS2 interface on MoS2 transistor performance: comparison of $\mathrm{Ag}$ and Ti contacts. ACS Appl Mater Interfaces. 2015;7:1180-7.

25. Li Q, Xiong HD, Liang X, Zhu X, Gu D, loannou DE, et al. Self-assembled nanowire array capacitors: capacitance and interface state profile. Nanotechnology. 2014;25:135201.

26. Millan NM, Lau P, Hann M, loannou D, Hoffman D, Barrionuevo M, et al. Hierarchical radial and polar organisation of chromosomes in human sperm. Chromosome Res. 2012;20:875-87. 
27. loannou D, Fonseka KG, Meershoek EJ, Thornhill AR, Abogrein A, Ellis M, et al. Twenty-four chromosome FISH in human IVF embryos reveals patterns of post-zygotic chromosome segregation and nuclear organisation. Chromosome Res. 2012;20:447-60.

28. Ioannou D, Meershoek EJ, Christopikou D, Ellis M, Thornhill AR, Griffin DK Nuclear organisation of sperm remains remarkably unaffected in the presence of defective spermatogenesis. Chromosome Res. 2011;19:741-53.

29. Ioannou D, Meershoek EJ, Thornhill AR, Ellis M, Griffin DK. Multicolour interphase cytogenetics: 24 chromosome probes, 6 colours, 4 layers. Mol Cell Probes. 2011;25:199-205.

30. Bourne G, Moir C, Bikkul U, Ahmed MH, Kill IR, Eskiw CH, et al. Interphase chromosome behavior in normal and diseased cells. Springer-Verlag New York: Springer; 2013

31. Tsirpanlis G, Bagos P, loannou D, Bleta A, Marinou I, Lagouranis A, et al. Exploring inflammation in hemodialysis patients: persistent and superimposed inflammation. A longitudinal study. Kidney Blood Press Res. 2004;27:63-70.

32. Bridger JM, Arican-Gotkas HD, Foster HA, Godwin LS, Harvey A, Kill IR, et al. The Non-random Repositioning of Whole Chromosomes and Individual Gene Loci in Interphase Nuclei and Its Relevance in Disease, Infection, Aging, and Cancer. Adv Exp Med Biol. 2014;773:263-79.

33. Mehta IS, Figgitt M, Clements CS, Kill IR, Bridger JM. Alterations to nuclear architecture and genome behavior in senescent cells. Ann N Y Acad Sci. 2007;1100:250-63.

34. Branco MR, Branco T, Ramirez F, Pombo A. Changes in chromosome organization during PHA-activation of resting human lymphocytes measured by cryo-FISH. Chromosome Res. 2008;16:413-26.

35. Skinner BM, Volker M, Ellis M, Griffin DK. An appraisal of nuclear organisation in interphase embryonic fibroblasts of chicken, turkey and duck. Cytogenet Genome Res. 2009;126:156-64.

36. Belmont A. Dynamics of chromatin, proteins, and bodies within the cell nucleus. Curr Opin Cell Biol. 2003;15:304-10.

37. Dion V, Gasser SM. Chromatin movement in the maintenance of genome stability. Cell. 2013;152:1355-64.

38. Branco MR, Pombo A. Intermingling of chromosome territories in interphase suggests role in translocations and transcription-dependent associations. PLoS Biol. 2006;4, e138.

39. Parada LA, McQueen PG, Misteli T. Tissue-specific spatial organization of genomes. Genome Biol. 2004:5:R44.

40. Naidoo N, Pawitan Y, Soong R, Cooper DN, Ku CS. Human genetics and genomics a decade after the release of the draft sequence of the human genome. Hum Genomics. 2011;5:577-622.

\section{Submit your next manuscript to BioMed Central and take full advantage of:}

- Convenient online submission

- Thorough peer review

- No space constraints or color figure charges

- Immediate publication on acceptance

- Inclusion in PubMed, CAS, Scopus and Google Scholar

- Research which is freely available for redistribution 